KÖDAI MATH. SEM. REP.

19 (1967), 410-414

\title{
THE SPHERICAL DERIVATIVE OF REGULAR AND MEROMORPHIC FUNCTIONS OF BOUNDED CHARACTERISTIC
}

\author{
By Masanobu Tsuzuki
}

1. Introduction. Let $D$ be the open unit disc in the complex plane. If $f(z)$ is a meromorphic function in $D$, we denote the spherical derivative of $f(z)$ by

$$
\rho(f(z))=\frac{\left|f^{\prime}(z)\right|}{1+|f(z)|^{2}} .
$$

Lehto and Virtanen, and Noshiro obtained the following results [2], [4].

Theorem A. A non-constant $f(z)$, meromorphic in $D$, is normal if and only if it satisfies an inequality

$$
\sup _{|z|<1}(1-|z|) \rho(f(z)) \leqq C,
$$

where $C$ is a finite constant.

CoRollary A. If (z) is normal meromorpluc in $D$, its characteristu function $T(r)$ fulfills the following relation:

$$
T(r)=0\left(\log \frac{1}{1-r}\right)
$$

Let $h(r)$ be a positive function such that $h(r)=o(r)(r \rightarrow 0)$. The connection between $\rho(f(z))$ and Picard's Theorem is shown by the following result of Gavrilov [1].

TheORLM B. Let $f(z)$ be meromorphic in $D$. If for a sequence $\left\{z_{n}\right\}, \lim _{n \rightarrow \infty}\left|z_{n}\right|=1$ and

$$
\lim _{n \rightarrow \infty} h\left[\left(1-\left|z_{n}\right|^{2}\right)\right] \rho\left(f\left(z_{n}\right)\right)=+\infty,
$$

then, Picard's Theorem holds for $f(z)$ in the union of any infinite subsequence of the discs

$$
D_{n}=\left\{z \in D \mid \sigma\left(z, z_{n}\right)<\varepsilon\left(1-\left|z_{n}\right|^{2}\right)^{-1} h\left[\left(1-\left|z_{n}\right|^{2}\right)\right]\right\},
$$

for each $\varepsilon>0$, where $\sigma\left(z, z_{n}\right)$ is the non-Euclidean hyperbolic distance between $z$ and $z_{n}$ in $D$.

Received March 23, 1967. 
The aim of this paper is to show that for regular functions in $D$ the boundedness of $T(r)$ imposes a restriction on the growth of $\rho(f(z))$, but does not for meromorphic functions.

The author expresses his heartiest thanks to Professors O. Ishikawa, M. Ozawa and N. Suita for their kind remarks in preparing this paper and to Mr. Hidenobu Yoshida for his valuable advice.

2. Theorem 1. Suppose that $f(z)$ is regular and of bounded type in D. Then

$$
(1-|z|) \rho(f(z)) \leqq e^{c /(1-|z|)},
$$

where $c$ is a positive constant.

The result (1) is sharp in such a sence that no improvement is possible on the order of $1 /(1-|z|)$ in $(1)$.

Theorem 2. Let $\varphi(r), 0 \leqq r<1$, denote any positive monolonically increasing function with

$$
\lim _{r \rightarrow 1} \varphi(r)=+\infty .
$$

Then, there exists a meromorphic function $f(z)$ of bounded type in I) such that

$$
\lim _{\substack{r \rightarrow 1 \\|z|=r}} \frac{1-|z|}{\varphi(r)} \rho(f(z))=+\infty .
$$

Proof of Theorem 1. By the condition of $f(z)$ we can assume that

$$
f(z)=\frac{\pi_{2}(z)}{\pi_{1}(z)}
$$

where $\pi_{i}(z)$ is regular, $\left|\pi_{i}(z)\right|<1,(i=1,2)$, and $\pi_{1}(z) \neq 0$. Schwar's's lemma yields inequalities

Hence

$$
\left|\pi_{\imath}{ }^{\prime}(z)\right| \leqq \frac{1}{1-|z|} \quad(i=1,2)
$$

$$
\left|\pi_{2}(z) \pi_{1}^{\prime}(z)\right| \leqq \frac{1}{1-|z|}, \quad\left|\pi_{2}^{\prime}(z) \pi_{1}(z)\right| \leqq \frac{1}{1-|z|} .
$$

On the other hand if $\pi(z)$ is regular and of bounded type in $D, \pi(z)$ satisfies

$$
|\pi(z)| \leqq e^{c /(1-|z|)},
$$

where $c$ is a fixed positive constant [5]. From (2) and (3)

$$
\begin{aligned}
(1-|z|) \rho(f(z)) & \leqq(1-|z|) \frac{\left|\pi_{2}(z) \pi_{1}{ }^{\prime}(z)\right|+\left|\pi_{2}{ }^{\prime}(z) \pi_{1}(z)\right|}{\left|\pi_{1}(z)\right|^{2}} \\
& \leqq e^{c /(1-|z|)},
\end{aligned}
$$


since $1 /\left(\pi_{1}(z)\right)^{2}$ is regular and of bounded type in $D$. We require a lemma to prove the latter of theorem 1 and theorem 2. Let

$$
x_{n}=1-e^{-n} \quad(n=1,2, \cdots) .
$$

Consider the Blaschke-product

$$
B(z)-\prod_{n=1}^{\infty} \frac{x_{n}-z}{1-x_{n} z}
$$

Lemma. For the product (4) we have

$$
\lim _{n \rightarrow \infty}\left(1-x_{n}\right)\left|B^{\prime}\left(x_{n}\right)\right| \geqq B,
$$

where $B$ is a positive constant.

Proof. Let

$$
B_{p}(z)=\prod_{n=1}^{p-1} \frac{x_{n}-z}{1-x_{n} z} \prod_{n=p+1}^{\infty} \frac{x_{n}-z}{1-x_{n} z}
$$

Then,

$$
B^{\prime}\left(x_{p}\right)=-\frac{1}{1-x_{p}^{2}} B_{p}\left(x_{p}\right)
$$

We write

$$
\left|B_{p}\left(x_{p}\right)\right|=\prod_{n=1}^{p-1} \frac{x_{p}-x_{n}}{1-x_{p} x_{n}} \prod_{n=p+1}^{\infty} \frac{x_{n}-x_{p}}{1-x_{p} x_{n}}
$$

Since, for $n=1,2, \cdots, p-1$

$$
\equiv T_{1}(p) T_{2}(p)
$$

$$
\frac{x_{p}-x_{n}}{1-x_{p} x_{n}}>\frac{1-e^{n-p}}{1+e^{n-p}},
$$

we infer that

$$
T_{1}(p)>\prod_{n=1}^{p-1} \frac{1-e^{-n}}{1+e^{-n}}
$$

and hence

$$
\lim _{p \rightarrow \infty} T_{1}(p) \geqq \prod_{n=1}^{\infty} \frac{1-e^{-n}}{1+e^{-n}} \equiv \Lambda>0 .
$$

Similarly,

$$
\lim _{p \rightarrow \infty} T_{2}(p) \geqq A .
$$

Therefore

$$
\lim _{p \rightarrow \infty}\left|B_{p}\left(x_{p}\right)\right| \geqq B>0 .
$$

By (6) this establishes (5). 
Completion of Theorem 1 . We form the function

$$
f(z)=B(z) e^{(1+z) /(1-z)} .
$$

$f(z)$ is evidently regular and of bounded type in $D$. For $p=1,2, \cdots$,

$$
f^{\prime}\left(x_{p}\right)=B^{\prime}\left(x_{p}\right) e^{\left(1+x_{p}\right) /\left(1-x_{p}\right)}
$$

and hence, from the lemma

$$
\lim _{p \rightarrow \infty} \frac{\left(1-x_{p}\right) \rho\left(f\left(x_{p}\right)\right)}{e^{(1+x p) /(1-x p)}} \geqq B>0 .
$$

Therefore we have the remainder in theorem 1 .

Proof of Theorem 2. Choose a natural number $K_{1}$ such that

$$
K_{1}^{2}\left|B^{\prime}\left(x_{1}\right)\right|\left\{1-\left(x_{1}+\frac{1}{K_{1}^{2}}\right) x_{1}\right\}>\varphi\left(x_{1}\right), e^{-1}>\frac{1}{K_{1}^{2}} .
$$

After natural numbers $K_{1}, K_{2}, \cdots, K_{p-1}$ are defined, we choose $K_{p}$ with inequalities

$$
K_{p}{ }^{2}\left|B^{\prime}\left(x_{p}\right)\right|\left\{1-\left(x_{p}+\frac{1}{K_{p}^{2}}\right) x_{p}\right\}>\varphi\left(x_{p}\right) \cdot p, \quad e^{-p}>\frac{1}{K_{p}^{2}},
$$

$$
K_{i}<K_{p}, \quad i=1,2, \cdots, p-1 .
$$

By this process we have a increasing sequence $\left\{K_{p}\right\}, p=1,2, \cdots$, satisfying (8). Let

$$
B_{1}(z)=\prod_{n=1}^{\infty} \frac{\left(x_{n}+1 / K_{n}^{2}\right)-z}{1-\left(x_{n}+1 / K_{n}^{2}\right) z}
$$

With $B(z)$ in the above lemma we form the function

$$
f(z)=\frac{B(z)}{B_{1}(z)} \text {. }
$$

It is obvious that $f(z)$ is meromorphic and of bounded type in $D$. Now,

Hence, from (8) we obtain

$$
\rho\left(f\left(x_{p}\right)\right)=\left|\frac{B^{\prime}\left(x_{p}\right)}{B_{1}\left(x_{p}\right)}\right| .
$$

and

$$
\rho\left(f\left(x_{p}\right)\right)>\varphi\left(x_{p}\right) \cdot p
$$

$$
\varlimsup_{\substack{z|\rightarrow 1 \\ z|=r}} \frac{\rho(f(z))}{\varphi(r)} \geqq \varlimsup_{p \rightarrow \infty} \frac{\rho\left(f\left(x_{p}\right)\right)}{\varphi\left(x_{p}\right)}=+\infty .
$$

This holds too, when we take as $\varphi(r), \varphi(r) /(1-r)$. 


\section{REFERENCES}

[1] Gavrilov, V. I., Meromorphic functions in the unit circle, whose spherical derivative has a given growth. Math. Sbor. 71 (1966), 387-404. (Russian)

[2] Lehto, O., AND K. I. ViRTANEn, Boundary behavior and normal meromorphic functions. Acta Math. 97 (1957), 47-56.

[ 3 ] Nevanlinna, R., Eindeutige analytische Funktionen. 2. Aufl., Springer-Verlag (1953).

[4] Noshiro, K., Contributions to the theory of meromorphic functions in the unit circle. J. Fac. Sc1. Hokkaido Unıv. 7 (1958), 149-159.

[5] Privalov, I. I., Randeigenschaften analytıscher Funktıonen. VEB Deutscher Verlag der Wissenschaften (1956).

Tokyo Metroporitan University. 